\title{
STABLY NON-SYNCHRONIZABLE MAPS OF THE PLANE
}

\author{
PATRICE LE CALVEZ, MARCO MARTENS, CHARLES TRESSER, AND PATRICK A. WORFOLK
}

\begin{abstract}
Pecora and Carroll presented a notion of synchronization where an $(n-1)$ dimensional nonautonomous system is constructed from a given $n$-dimensional dynamical system by imposing the evolution of one coordinate. They noticed that the resulting dynamics may be contracting even if the original dynamics are not. It is easy to construct flows or maps such that no coordinate has synchronizing properties, but this cannot be done in an open set of linear maps or flows in $\mathbb{R}^{n}, n \geq 2$. In this paper we give examples of real analytic homeomorphisms of $\mathbb{R}^{2}$ such that the non-synchronizability is stable in the sense that in a full $C^{0}$ neighborhood of the given map, no homeomorphism is synchronizable.
\end{abstract}

\section{INTRODUCTION}

The observation that nonlinear oscillators can synchronize when coupled was first reported in a letter that Christiaan Huyghens wrote to his father Huy. This phenomenon has inspired many investigations by physicists and mathematicians over the years. Another (although related) kind of synchronization has recently received much attention because of its potential applications, and because of the surprise caused by its discovery by Pecora and Carroll [PC1]. Roughly speaking, they noticed that if one constructs an $(n-1)$-dimensional nonautonomous evolution equation from a given $n$-dimensional one by imposing the evolution of one coordinate, the resulting dynamics could be contracting, even when the original dynamics are not. The surprise was that this works for chaotic evolution equations such as the Lorenz equations as observed in [PC1] and subsequently proved in [HV]. We first recall what we need from [PC1] and [PC2].

Consider the Lorenz equations:

$$
\left\{\begin{array}{l}
\dot{x}=\sigma(y-x), \\
\dot{y}=r x-y-x z, \\
\dot{z}=x y-b z .
\end{array}\right.
$$

Pecora and Carroll [PC1] noticed that for any solution $(x(t), y(t), z(t))$ of these equations, all solutions $(Y(t), Z(t))$ of the nonautonomous system

$$
\left\{\begin{array}{l}
\dot{Y}=r x(t)-Y-x(t) Z \\
\dot{Z}=x(t) Y-b Z
\end{array}\right.
$$

satisfy

$$
\lim _{t \rightarrow+\infty}|y(t)-Y(t)|=\lim _{t \rightarrow+\infty}|z(t)-Z(t)|=0 .
$$

They called this phenomenon synchronization, and the name master-slave synchronization was proposed in [TWB to avoid confusion with other, previously recognized, phenomena. 
More is true for the Lorenz system: for any smooth function $x(t)$, not necessarily a solution of the Lorenz equations,

$$
\lim _{t \rightarrow+\infty}\left|Y_{1}(t)-Y_{2}(t)\right|=\lim _{t \rightarrow+\infty}\left|Z_{1}(t)-Z_{2}(t)\right|=0
$$

for any two initial conditions $\left(Y_{1}(0), Z_{1}(0)\right),\left(Y_{2}(0), Z_{2}(0)\right)$ of the above nonautonomous system. This stronger property was called absolute (master-slave) synchronization in [TWB where all these concepts were defined in a more geometric and coordinate independent way. Since the only kind of synchronization we will be dealing with in this paper is of the masterslave kind, we shall from now on omit this qualification. Synchronization also occurs for maps, and this will be our sole concern in this paper. Accordingly we next recall the relevant definitions from [TWB].

Let $\mathcal{M}$ be a manifold of dimension greater than one and let $F: \mathcal{M} \rightarrow \mathcal{M}$ be a mapping. Assume that $\mathcal{M}$ has a product structure so that $\mathcal{M}=M \times N$ with $M, N$ manifolds of dimension at least one, and $\operatorname{dim} M=m$. By a choice of product structure on $\mathcal{M}$, we mean a choice of a pair of foliations $\mathcal{F}$ and $\mathcal{G}$ such that each leaf of $\mathcal{F}$ is homeomorphic to $M$, each leaf of $\mathcal{G}$ is homeomorphic to $N$, and each leaf of $\mathcal{F}$ crosses each leaf of $\mathcal{G}$ at a single point. If we choose any leaf $\mathcal{F}_{0}$ in $\mathcal{F}$, we can now identify $\mathcal{F}_{0}$ with $M$ and define the map $\Pi_{M}: \mathcal{M} \rightarrow M$ as the projection onto $\mathcal{F}_{0}$ along $\mathcal{G}$. Similarly, the choice of a leaf $\mathcal{G}_{0}$ in $\mathcal{G}$ and an identification of $\mathcal{G}_{0}$ with $N$ define a map $\Pi_{N}: \mathcal{M} \rightarrow N$. We could as well think in terms of choices of coordinate system and call $\Pi_{M}(p)$ and $\Pi_{N}(p)$ the $x$ and $y$ coordinates of $p \in \mathcal{M}$. For any choice of product structure, we can perform the following construction to produce a nonautonomous mapping on the second factor. Let $\{x(i)\}_{i=0}^{\infty}$ be a sequence of points in $M$ (in practice, this sequence can be defined as the successive $x$-coordinates of a sequence of points $\{p(i)\}_{i=0}^{\infty}$ in $\left.\mathcal{M}\right)$. Define $\tilde{F}_{x}: \mathbb{N} \times N \rightarrow N$ by

$$
\tilde{F}_{x}(n, y)=\Pi_{N} F(x(n), y) .
$$

A nonautonomous mapping on $N$ is defined by

$$
Y(n+1)=\tilde{F}_{x}(n, Y(n)) .
$$

With $d_{N}$ standing for the distance on $N$, we say the system $(\mathcal{M}, F, \mathcal{F}, \mathcal{G})$ is absolutely $m$ synchronizing if for all sequences $\{x(i)\}_{i=0}^{\infty}$, the motions under $\tilde{F}_{x}$ on $N$ of all initial conditions converge, i.e., for all $Y_{1}(0), Y_{2}(0)$,

$$
\lim _{n \rightarrow \infty} d_{N}\left(Y_{1}(n), Y_{2}(n)\right)=0 .
$$

If the convergence does not necessarily occur for all sequences $\{x(i)\}_{i=0}^{\infty}$, but does occur whenever the sequence $\{p(i)\}_{i=0}^{\infty}$ is defined as the successive points of an orbit of $F$, we say the system $(\mathcal{M}, F, \mathcal{F}, \mathcal{G})$ is $m$-synchronizing. Notice that with $p(i)=(x(i), y(i))$, for all $Y(0)$ we then have $\lim _{n \rightarrow \infty} d_{N}(Y(n), y(n))=0$, which means that $Y$ synchronizes to $y$.

The map $F$ is (absolutely) $m$-synchronizable if a product structure can be exhibited on $\mathcal{M}$ such that the system $(\mathcal{M}, F, \mathcal{F}, \mathcal{G})$ is (absolutely) $m$-synchronizing. When $m=1$, the $m$-prefix will usually be omitted. For instance, any Hénon map is absolutely synchronizable; in the standard coordinate system, such a map is given by an equation of the form $F(u, v)=$ 
$\left(v, 1-a v^{2}+b u\right)$ so that if one imposes the time evolution of $v$ then the evolution of $u$ is independent of $u(0)\left(\right.$ since $\left.u(n+1)=\tilde{F}_{v}(n, u(n))=v(n)\right)$.

One can have good reasons to consider product structures where the foliations $\mathcal{F}$ and $\mathcal{G}$ have some degree of smoothness, or some other structure: for instance, particular attention is given in [TWB to linear foliations in the case when $\mathcal{M}=\mathbb{R}^{d}$ and $F$ is a linear map. Note that instead of changing the product structure, one can equivalently keep the product structure fixed and consider all maps conjugated to $F$, where the smoothness of the conjugacy is the same as the smoothness of the foliations corresponding to the product structures one wants to consider. The following result is obtained in [TWB].

Theorem 1 ( [TWB]). Consider the synchronizing systems $\left(\mathbb{R}^{d}, F, \mathcal{F}, \mathcal{G}\right)$ where $d \geq 2, F$ is a linear map or flow, $\mathcal{F}$ is the foliation by lines where $x_{i}$ is constant for $i>1$, and $\mathcal{G}$ is the foliation by planes $x_{1}=$ constant. Then the set of maps or flows linearly conjugate to such an $F$ form open and dense sets among linear maps and flows in $\mathbb{R}^{d}$.

This theorem admits the following corollary.

Corollary 1 ([TWB]). The linear maps and flows in $\mathbb{R}^{d}, d \geq 2$, which are absolutely synchronizable form open and dense subsets of the linear maps and flows on these spaces.

Remark. Consider the system $(\mathcal{M}, F, \mathcal{F}, \mathcal{G})$. Suppose the map $F$ has a fixed point $q_{0}$. If we consider the orbit $p(i) \equiv q_{0}$, then the map $\tilde{F}_{x}$ is an autonomous map from the leaf $\mathcal{F}_{0}$ containing $q_{0}$ to itself. Clearly, $q_{0}$ is a fixed point of this map. If $\tilde{F}_{x}$ has another fixed point, then the system $(\mathcal{M}, F, \mathcal{F}, \mathcal{G})$ is not synchronizing. If this holds true for all pairs of foliations $\mathcal{F}, \mathcal{G}$, then $F$ is not synchronizable.

Using this remark, it is easy to find examples of diffeomorphisms of $\mathbb{R}^{d}, d \geq 2$, which are not synchronizable. The identity, and more generally, any map which leaves the boundary of a topological $d$-ball pointwise invariant are such maps. However, there are arbitrarily small perturbations (in any reasonable metric) of these examples which lose this property. In view of Theorem 1, this leads to the question of whether non-synchronizability can occur in a robust way. To make this question more precise, we formulate the following

Definition. A map is $k$-stably non-synchronizable if it is $C^{k}$ and every map sufficiently $C^{k}$ close to it is non-synchronizable. A homeomorphism is k-stably non-synchronizable if it is $C^{k}$ and every homeomorphism sufficiently $C^{k}$ close to it is non-synchronizable. When $k=0$, the $k$ prefix will be omitted.

Examples of linear stably non-synchronizable maps on the two-dimensional torus $\mathbb{T}^{2}$ were given in TWB (a more comprehensive treatment of this matter, including necessary and sufficient conditions for synchronizability for automorphisms of $\mathbb{T}^{d}$, will be given elsewhere [MTW]). The situation in Euclidean spaces is quite different. So far, we could not find any pair $(r, k), k \leq r$ for which we could exhibit a $C^{r}$ map in $\mathbb{R}^{d}, d \geq 3$ which is $k$-stably non-synchronizable. To the contrary in $\mathbb{R}^{2}$, we shall prove

Theorem 2. There exist real-analytic stably non-synchronizable homeomorphisms of $\mathbb{R}^{2}$. 


\section{Proof of Theorem 2}

We denote by $\mathbb{T}^{1}=\mathbb{T}$ the one-dimensional torus, and $\pi_{1}, \pi_{2}$ the standard projections defined on the plane $\mathbb{R}^{2}$ or the annulus $\mathbb{A}=\mathbb{T} \times[0,1]$ (this ambiguity should not cause any confusion). Recall that a simple closed curve $C \subset \mathbb{A}$ is essential if it is not null homotopic. The curve $C^{\prime} \subset A$ is above (respectively, below) $C$ if it lies in the same component of $\mathbb{A} \backslash C$ as $\mathbb{T} \times\{1\}$ (respectively, $\mathbb{T} \times\{0\}$ ). Finally, two points $x$ and $y$ in a topological space $S$ are separated by a subset $T \subset S$ if they lie in different connected components of $S \backslash T$.

Lemma 1. Let $F$ be a homeomorphism of $\mathbb{A}$ isotopic to the identity (i.e., orientation preserving and leaving each boundary circle invariant) which satisfies the following conditions:

i) There exists an essential simple closed curve $C \subset \mathbb{T} \times(0,1)$ such that $F(C)$ is disjoint from $C$ and is above $C$, and such that the sequence $\left(F^{n}(C)\right)_{n>0}$ accumulates on $\mathbb{T} \times\{1\}$ and the sequence $\left(F^{-n}(C)\right)_{n>0}$ accumulates on $\mathbb{T} \times\{0\}$.

ii) There exists a lift $f$ of $F$ to the universal cover $\tilde{\mathbb{A}}=\mathbb{R} \times[0,1]$ of $\mathbb{A}$ such that

$$
\text { for all } x \in \mathbb{R}\left\{\begin{array}{l}
\pi_{1} \circ f(x, 0)<x, \\
\pi_{1} \circ f(x, 1)>x .
\end{array}\right.
$$

Then for each simple arc $\gamma:[0,1] \rightarrow \tilde{\mathbb{A}}$ such that $\gamma(0) \in \mathbb{R} \times\{0\}, \gamma(1) \in \mathbb{R} \times\{1\}$, $\gamma((0,1)) \subset \mathbb{R} \times(0,1)$, there exists $t$ and $t^{\prime}$ in $(0,1)$ with $t^{\prime}>t$ such that

$$
f(\gamma(t))=\gamma\left(t^{\prime}\right) \text {. }
$$

Proof of Lemma 1. We want first to construct a simple arc $\lambda:[0,1] \rightarrow \tilde{\mathbb{A}}$ which satisfies the conclusion of the lemma and has the additional property that there does not exist $u$ and $u^{\prime}$ in $(0,1)$ with $u^{\prime}<u$ such that $f(\lambda(u))=\lambda\left(u^{\prime}\right)$.

A change of variables may be made in $\mathbb{R} \times(0,1)$ such that each $f^{k}(C), k \in \mathbb{Z}$, is of the form $\mathbb{T} \times\left\{r_{k}\right\}$. It follows that $\pi_{2}^{\prime}(F(z))>\pi_{2}^{\prime}(z)$ for each $z \in \mathbb{T} \times(0,1)$, where $\pi_{2}^{\prime}$ is the second projection with respect to the new coordinate system. This fact combined with property $i i)$ implies that $f$ has no fixed point in $\tilde{\mathbb{A}}$. Let $C_{\epsilon}$ and $C_{1-\epsilon}$ be the curves which in the new coordinate system are given by, respectively, $\mathbb{R} \times\{\epsilon\}$ and $\mathbb{R} \times\{1-\epsilon\}$. The curve $C_{\epsilon}$ is pointwise close in the original coordinates to the boundary component $\mathbb{R} \times\{0\}$ of $\tilde{\mathbb{A}}$, and similarly for $C_{1-\epsilon}$ to $\mathbb{R} \times\{1\}$. We now choose one of the points $p$ of $C_{\epsilon}$ closest to $\mathbb{R} \times\{0\}$ and $q$ of $C_{1-\epsilon}$ closest to $\mathbb{R} \times\{1\}$. We define $\lambda$ by sending $[0, \epsilon]$ to the vertical segment in $\tilde{\mathbb{A}}$ joining $\mathbb{R} \times\{0\}$ to $p$, by sending $[1-\epsilon, 1]$ to the vertical segment in $\tilde{\mathbb{A}}$ joining $q$ to $\mathbb{R} \times\{1\}$, and sending $[\epsilon, 1-\epsilon]$ to any arc joining $p$ to $q$ so that $\pi_{2}^{\prime} \circ \lambda$ is strictly increasing on $[\epsilon, 1-\epsilon]$. In the previous sentence, vertical is with respect to the original coordinate system. See Figure 1. By property $i i)$ there exist $t$ and $t^{\prime}$ in $(0,1)$ such that

$$
f(\lambda(t))=\lambda\left(t^{\prime}\right),
$$

and by the property of $\pi_{2}^{\prime}$ necessarily $t^{\prime}>t$, if $\epsilon$ is chosen small enough.

Let $\lambda$ be the arc we just constructed and let $\gamma$ be the arc from the statement of Lemma 1. We can construct a continuous function

$$
\Gamma:[0,1] \times[0,1] \rightarrow \tilde{\mathbb{A}},
$$




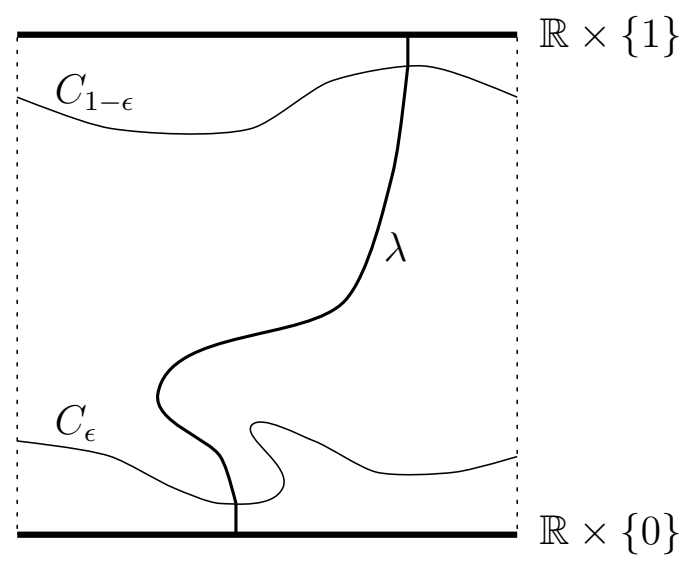

Figure 1. The curves $C_{\epsilon}$ and $C_{1-\epsilon}$ and the arc $\lambda$ in the original coordinate system. such that, with $\Gamma(s, t)=\gamma_{s}(t)$, we have for each $s$ and $t$ in $[0,1]$,

$$
\left\{\begin{array}{c}
\gamma_{0}(t)=\lambda(t), \\
\gamma_{1}(t)=\gamma(t), \\
\gamma_{s}(0) \in \mathbb{R} \times\{0\}, \\
\gamma_{s}(1) \in \mathbb{R} \times\{1\}, \\
\gamma_{s}(t) \in \mathbb{R} \times(0,1) \text { if } t \in(0,1) .
\end{array}\right.
$$

We can write $[0,1]^{2}=U^{+} \sqcup U^{-} \sqcup K$ where

$$
\begin{gathered}
U^{-}=\left\{(s, t) \mid f\left(\gamma_{s}(t)\right) \text { is to the left of } \gamma_{s}([0,1])\right\} \\
U^{+}=\left\{(s, t) \mid f\left(\gamma_{s}(t)\right) \text { is to the right of } \gamma_{s}([0,1])\right\}, \\
K=\left\{(s, t) \mid f\left(\gamma_{s}(t)\right) \in \gamma_{s}([0,1])\right\} .
\end{gathered}
$$

Note that $U^{-}$and $U^{+}$are open subsets of $[0,1]^{2}$ which respectively contain $[0,1] \times\{0\}$ and $[0,1] \times\{1\}$, and that $K$ is a closed subset of $[0,1]^{2}$.

We can define a map $\phi: K \rightarrow[0,1]$ by setting

$$
f\left(\gamma_{s}(t)\right)=\gamma_{s}(\phi(s, t)) \text { if }(s, t) \in K .
$$

This map is continuous and, since $f$ has no fixed point, we can write

$$
K=K^{+} \sqcup K^{-},
$$

where $K^{+}$and $K^{-}$are closed subsets of $K$ defined by

$$
\begin{aligned}
& K^{+}=\{(s, t) \in K \mid \phi(s, t)>t\}, \\
& K^{-}=\{(s, t) \in K \mid \phi(s, t)<t\} .
\end{aligned}
$$

Let us remark that

$$
\left\{\begin{array}{l}
K^{+} \cap\{0\} \times[0,1] \neq \emptyset, \\
K^{-} \cap\{0\} \times[0,1]=\emptyset
\end{array}\right.
$$


due to the properties of $\lambda$.

To prove Lemma 1, we have to show that

$$
K^{+} \cap\{1\} \times[0,1] \neq \emptyset .
$$

Assume the contrary holds true. Then there exists $\epsilon>0$ such that

$$
\left\{\begin{array}{c}
K^{+} \cap[1-\epsilon, 1] \times[0,1]=\emptyset, \\
K^{-} \cap[0, \epsilon] \times[0,1]=\emptyset,
\end{array}\right.
$$

and such that

$$
\left\{\begin{array}{c}
{[0,1] \times[1-\epsilon, 1] \subset U^{+}} \\
{[0,1] \times[0, \epsilon] \subset U^{-}}
\end{array}\right.
$$

We set $x=\left(\frac{1}{2}, 1-\epsilon\right) \in U^{+}, y=\left(\frac{1}{2}, \epsilon\right) \in U^{-}$and we denote by $\Delta$ the boundary of the square $[0,1]^{2}$. Now:

- the points $x$ and $y$ are separated neither by the closed set $\Delta \cup K^{-}$, nor by the closed set $\Delta \cup K^{+}$,

- the intersection of $\Delta \cup K^{-}$and $\Delta \cup K^{+}$is $\Delta$, which is a connected set.

By Alexander's Lemma (see [New, Theorem 9.2]), we deduce from these two facts that $x$ and $y$ are not separated by $\left(\Delta \cup K^{-}\right) \cup\left(\Delta \cup K^{+}\right)=\Delta \cup K$. This is clearly impossible because $x \in U^{+}$and $y \in U^{-}$.

Returning from working on the universal cover to working on the annulus, we deduce from Lemma 1 the following corollary.

Corollary 2. Under the assumptions of Lemma $[1$, if $\gamma:[0,1] \rightarrow \mathbb{A}$ is an arc starting at $\mathbb{T} \times\{0\}$, terminating at $\mathbb{T} \times\{1\}$, and taking its intermediate values in $\mathbb{T} \times(0,1)$, then there exist $t$ and $t^{\prime}$ in $(0,1)$ with $t^{\prime}>t$ such that $F(\gamma(t))=\gamma\left(t^{\prime}\right)$.

If $F$ satisfies the conditions of Lemma 1, we shall say that it is of type $(\mathrm{P})$. We shall say $F$ is of type $(\mathrm{Q})$ if it is of type $(\mathrm{P})$ and also satisfies the following condition iii) which is stronger than condition $i$ ) of Lemma 1 .

iii) There exists a lift $f$ of $F$ to the universal cover $\tilde{\mathbb{A}}=\mathbb{R} \times[0,1]$ of $\mathbb{A}$ such that for all $x \in \mathbb{R}$,

$$
\left\{\begin{array}{l}
\pi_{1} \circ f(x, 0)<x-1 \\
\pi_{1} \circ f(x, 1)>x+1
\end{array}\right.
$$

It is more convenient to present the following fact for the annulus than for the universal cover.

Lemma 2. Assume $F$ is of type (Q). If $\gamma$ and $\gamma^{\prime}$ are two disjoint arcs starting at $\mathbb{T} \times\{0\}$, terminating at $\mathbb{T} \times\{1\}$, and taking their intermediate values in $\mathbb{T} \times(0,1)$, we have

$$
F(\gamma([0,1])) \cap \gamma^{\prime}([0,1]) \neq \emptyset .
$$




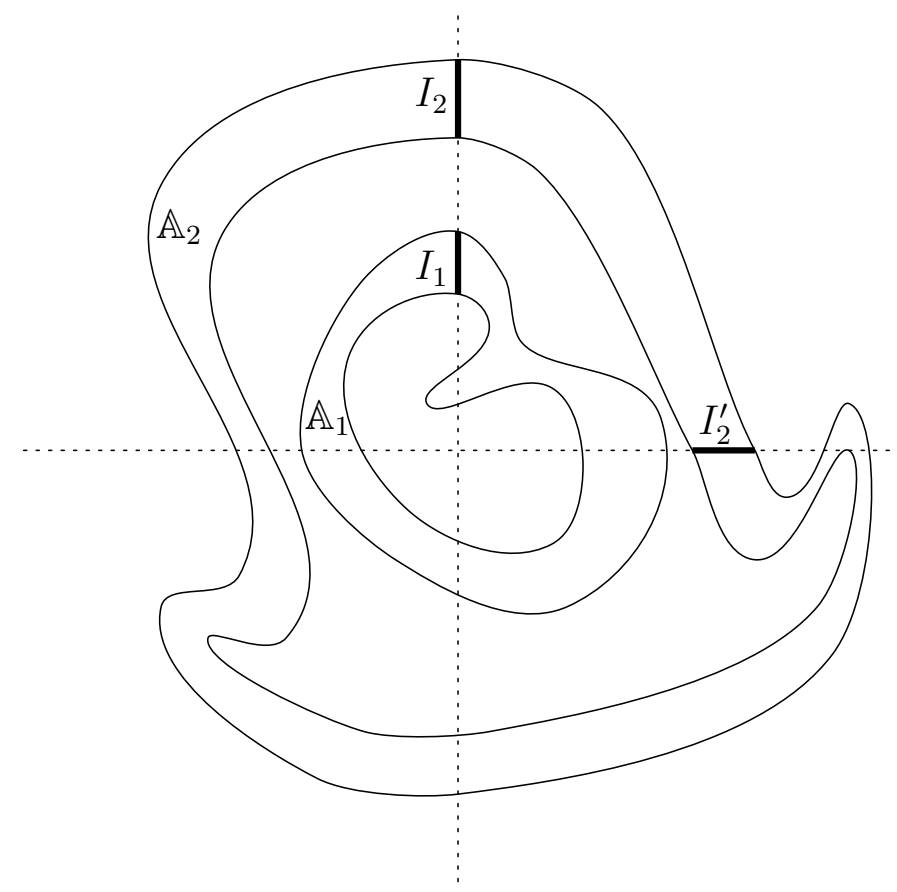

Figure 2. The annuli $\mathbb{A}_{1}$ and $\mathbb{A}_{2}$ and the intervals $I_{1}, I_{2}$, and $I_{2}^{\prime}$.

The proof is straightforward.

The next lemma gives a sufficient condition for non-synchronizability. Let us consider an orientation preserving homeomorphism $F$ of $\mathbb{R}^{2}$ which has two compact invariant annuli $\mathbb{A}_{1}$ and $\mathbb{A}_{2}$ such that $\mathbb{A}_{1}$ is included in the bounded component of $\mathbb{R}^{2} \backslash \mathbb{A}_{2}$. Assume furthermore that each of $\left.F\right|_{\mathbb{A}_{1}}$ and $\left.F\right|_{\mathbb{A}_{2}}$ is conjugated to a homeomorphism of type (Q) by a conjugacy which sends the inner boundary of $\mathbb{A}_{i}$ to $\mathbb{T} \times\{0\}$. We say such an $F$ satisfies condition $(\mathrm{R})$.

Lemma 3. If $F$ satisfies condition $(R)$, then it is not synchronizable.

Proof of Lemma 3. By the Brouwer Fixed Point Theorem, $F$ has a fixed point $z_{0}$ in the closure of the bounded connected component of $\mathbb{R}^{2} \backslash \mathbb{A}_{1}$. We can assume that $z_{0}=(0,0)$. We define

$$
\gamma:[0,+\infty) \rightarrow \mathbb{R}, \quad t \mapsto(0, t)
$$

and

$$
\gamma^{\prime}:[0,+\infty) \rightarrow \mathbb{R}, \quad t \mapsto(t, 0)
$$

It is easy to see that there exist two disjoint compact intervals $I_{1}$ and $I_{2}$ of $[0,+\infty)$, the first one to the left of the second one, such that $\left.\gamma\right|_{I_{i}}$ starts at the inner boundary of $\mathbb{A}_{i}$, ends at the upper boundary of $\mathbb{A}_{i}$, and takes its intermediate values in the interior of $\mathbb{A}_{i}$. Similarly, there exists a compact interval $I_{2}^{\prime} \subset(0,+\infty)$ such that $\left.\gamma^{\prime}\right|_{I_{2}^{\prime}}$ satisfies the same condition as $\left.\gamma\right|_{I_{2}}$. See Figure 2. By Corollary 2 there exist $t_{1}$ and $t_{2}$ in $I_{1}$ with $t_{1}<t_{2}$, and by Lemma 2 
there exists $t_{3}$ in $I_{2}$ (thus $\left.t_{3}>t_{2}\right)$ such that

$$
\left\{\begin{array}{l}
F\left(\gamma\left(t_{1}\right)\right)=\gamma\left(t_{2}\right) \\
F\left(\gamma\left(t_{3}\right)\right) \in \gamma^{\prime}\left(I_{2}^{\prime}\right)
\end{array}\right.
$$

It follows that the continuous map $\psi: t \mapsto \pi_{2}(F(0, t))$ satisfies $\psi\left(t_{1}\right)>t_{1}$ and $\psi\left(t_{3}\right)=0$. Consequently, $\psi$ has a fixed point between $t_{1}$ and $t_{3}$ as well as another fixed point at 0 . With the notation of the first section, the map $\psi$ is the same as $\tilde{F}_{x}$ with $x(i) \equiv 0$. From the remark in the first section, $F$ is not synchronizing. Since condition $(R)$ is stable under conjugacy, $F$ is not synchronizable.

Proof of Theorem 8 . Consider now a real analytic homeomorphism $F: \mathbb{R}^{2} \rightarrow \mathbb{R}^{2}$ given in polar coordinates by

$$
(\theta, r) \mapsto(\theta+\beta(r), \alpha(r))
$$

We further assume that the map $\alpha:[0, \infty) \rightarrow[0, \infty)$ has fixed points at $r=0,1,2,3$, 4 such that 0,2 , and 4 are sinks, while 1 and 3 are sources.

We denote by $S_{r}$ the disk with center $O$ and radius $r$. Let $\mathbb{A}_{1}$ be the annulus between $S_{1}$ and $S_{2}$ and $\mathbb{A}_{2}$ be the annulus between $S_{3}$ and $S_{4}$. Finally, we assume that $\left.F\right|_{\mathbb{A}_{1}}$ and $\left.F\right|_{\mathbb{A}_{2}}$ are of type $(\mathrm{Q})$, so that $F$ satisfies condition $(\mathrm{R})$. An example of a map $F$ satisfying all these conditions is obtained by taking

$$
\begin{aligned}
& \alpha(r)=r+\mu r\left(r^{2}-1\right)\left(r^{2}-4\right)\left(r^{2}-9\right)\left(r^{2}-16\right)\left(r^{2}-25\right), \\
& \beta(r)=2 \pi r^{2},
\end{aligned}
$$

with $\mu$ positive but sufficiently small.

If $G$ is a homeomorphism $C^{0}$ close to $F$, it does not necessarily satisfy condition (R) since it might lose the invariant curves. However we shall see $G$ is not synchronizable, hence $F$ is stably non-synchronizable. (One could check that any map close to $F$ in the $C^{1}$ topology does satisfy condition (R) and thus get, by Lemma 3, an independent proof of the fact that $F$ is $C^{1}$-stably non-synchronizable.)

In the rest of the discussion, proximity refers to the $C^{0}$ norm. If $G$ is close enough to $F$, the curve $G\left(S_{3 / 2}\right)$ is disjoint from $S_{3 / 2}$ and situated in the unbounded component of $\mathbb{R}^{2} \backslash S_{3 / 2}$. For any $n \geq 1$, we can consider the open annulus $V_{n}$ with boundaries $G^{-n}\left(S_{3 / 2}\right)$ and $G^{n}\left(S_{3 / 2}\right)$; these annuli form an increasing sequence and the set $V=\cup_{n \geq 1} V_{n}$ is an open annulus (i.e., homeomorphic to $\mathbb{T} \times(0,1)$ ) invariant under $G$.

Let us fix $\epsilon \in\left(0, \frac{1}{2}\right)$. If $G$ is close enough to $F$, the curves $S_{1-\epsilon}$ and $S_{2+\epsilon}$ are disjoint from their images under $G$, which are located in the same component, respectively of $\mathbb{R}^{2} \backslash S_{1-\epsilon}$ and $\mathbb{R}^{2} \backslash S_{2+\epsilon}$ as the images under $F$. The annulus $V$ is thus bounded between the curves $G\left(S_{1-\epsilon}\right)$ and $G\left(S_{2+\epsilon}\right)$. Furthermore, for $G$ close enough to $F, V$ contains the annulus bounded by $S_{1+\epsilon}$ and $S_{2-\epsilon}$. Proceeding between $S_{3}$ and $S_{4}$ as we just did between $S_{1}$ and $S_{2}$, we get a second open invariant annulus $W$.

By the Brouwer Fixed Point Theorem, we also know that $G$ admits a fixed point $z_{0}$ in the closed disk bounded by $S_{1-\epsilon}$. Thus we can define two maps from $[0, \infty)$ to $\mathbb{R}^{2}$ :

$$
\gamma: t \mapsto z_{0}+(0, t)
$$




$$
\gamma^{\prime}: t \mapsto z_{0}+(t, 0)
$$

and as before, we know that there exist two open intervals $I_{1}$ and $I_{2}$ with disjoint and compact closures, with $I_{1}$ to the left of $I_{2}$ such that $\left.\gamma\right|_{I_{1}}$ and $\left.\gamma\right|_{I_{2}}$ have value respectively in $V$ and $W$ and join the interior end to the exterior end of these annuli. Similarly, we know that there exists an open interval $I_{2}^{\prime}$ such that $\left.\gamma^{\prime}\right|_{I_{2}^{\prime}}$ has the same property as $\left.\gamma\right|_{I_{1}}$.

We now use Caratheodory's prime ends theory (see, e.g., [For, pp. 187-198]). We can compactify each connected piece of the boundary of $V$ as a circle, and extend $\left.G\right|_{V}$ as a map $\hat{G}$ on the compact annulus $\hat{V}$ obtained by this compactification. Notice that $\hat{G}$ satisfies condition $i$ ) with $C=S_{3 / 2}$. The interpretation of the prime ends as accessible points tells us that the rotation numbers of $\hat{G}$ on the boundary circles of $\hat{V}$ approach the corresponding rotation numbers of $\left.F\right|_{S_{1}}$ and $\left.F\right|_{S_{2}}$ when $G$ converges to $F$; in particular, $\hat{G}$ satisfies property iii) if $G$ is close enough to $F$.

We have a similar situation for $W$. Furthermore, we know that the $\left.\operatorname{arcs} \gamma\right|_{I_{1}},\left.\gamma\right|_{I_{2}}$, and $\left.\gamma^{\prime}\right|_{I_{2}^{\prime}}$ have limits at both ends which belong to the boundary circles of $\hat{V}$ or $\hat{W}$ that these arcs approach (this is a property of accessible points). Thus we can apply Corollary 2 and Lemma 2, and get the existence of $t_{1}<t_{2}<t_{3}$ such that

$$
\left\{\begin{array}{l}
\hat{G}\left(\gamma\left(t_{1}\right)\right)=\gamma\left(t_{2}\right), \\
\hat{G}\left(\gamma\left(t_{3}\right)\right) \in \gamma^{\prime}\left(I_{2}^{\prime}\right),
\end{array}\right.
$$

from which it follows that

$$
\left\{\begin{array}{l}
G\left(\gamma\left(t_{1}\right)\right)=\gamma\left(t_{2}\right), \\
G\left(\gamma\left(t_{3}\right)\right) \in \gamma^{\prime}\left(I_{2}^{\prime}\right) .
\end{array}\right.
$$

We thus conclude that $G$ is not synchronizable.

\section{REFERENCES}

[For] L.R. Ford. Automorphic Functions. Chelsea Publishing Co., New York, second edition, 1951.

[Huy] C. Huyghens. Letter to his father dated February 26, 1665, in Euvres Completes de Christian Huyghen, edited by M. Nijhoff, Société Hollandaise des Sciences, The Hagues, Netherlands, Vol.5, pp. 243-244, item no. 1335, 1893.

[HV] R. He and P. G. Vaidya. Analysis and synthesis of synchronous periodic and chaotic systems. Phys. Rev. A, 46:7387-7392, 1992.

[MTW] M. Martens, C. Tresser, and P. A. Worfolk. Synchronization of linear maps on euclidean spaces and tori. In preparation.

[New] M. H. A. Newman. Elements of the Topology of Plane Sets of Points. Cambridge University Press, Cambridge, 1951.

[PC1] L. M. Pecora and T. L. Carroll. Synchronization in chaotic systems. Phys. Rev. Lett., 64:821-824, 1990.

[PC2] L. M. Pecora and T. L. Carroll. Driving systems with chaotic signals. Phys. Rev. A, 44:2374-2383, 1991.

[TWB] C. Tresser, P. A. Worfolk, and H. Bass. Master-slave synchronization from the point of view of global dynamics. CHAOS, 5:693-699, 1995. 
URA 742 du CNRS, Institut Galilée, Université Paris 13, Avenue J.-B. Clément, 93420 Villetaneuse, France.

E-mail address: lecalvez@math.univ-paris13.fr

Institute of Mathematical Sciences, SUNy at Stony Brook, Stony Brook, Ny 11794-3651.

E-mail address: marco@math. sunysb.edu

I.B.M., P.O. Box 218, Yorktown Heights, NY 10598.

E-mail address: tresser@watson.ibm.com

The Geometry Center, 1300 S. Second St., Minneapolis, Mn 55454.

E-mail address: worfolk@geom.umn.edu 